C A S E

OF

\title{
CYSTIC DISEASE OF THE KIDNEY
}

\author{
SIMULATING OVARIAN DISEASE.
}

BY

HENRY COOPER ROSE, M.D., F.G.S., F.L.S.

Received F'eb. 27th.-Read May 12th, 1868.

Ат a period when the subject of ovariotomy is engaging the attention of surgeons in all parts of Europe, every case which tends to throw light upon the matter, either in rendering the diagnosis clear, or in pointing out difficulties to be overcome, cannot fail to be of interest. I do not hesitate, therefore, to draw attention to the following history as one capable of conveying some useful lessons.

C. R-, a somewhat delicately constituted young lady, æt. 18, came under my professional care in January, 1856. Menstruation had commenced at the age of thirteen, but had never been regular, there being intervals of three or four months in the catamenial periods. Two years before (in 1854) she had observed blood in her urine, but had never spoken of it. This symptom continued occasionally for 
some years. She stated that a swelling had existed low down in the left side in the inguinal region for some time.

On examination $I$ found a tumour occupying the position of the left ovary about the size of a large orange. In the following May there was tenderness over the left side, extending from the edge of the false ribs to the inguinal region, with a distinct prominence. The uterus was moveable and normal in size. The tumour which filled the space appeared to be solid-no fluctuation was perceptible. Her general health had not suffered much. In the September following, the tumour was larger, and there was a sense of deep fluctuation, but this was questionable. Hæmaturia had been more frequent, and sometimes pain of a dull, aching, character was complained of. Gallic acid always relieved the hæmaturia; and she had continued to menstruate as before, every two or three months.

From this time until November, 1858 (a period of two years), she had been much better, the turnour remaining very much in the same state. Now, however, she had a severe attack of hæmaturia, which weakened her considerably.

The tumour now occupied the whole region from the diaphragm to the pubes and Poupart's ligament of the left side. It was very prominent a little to the left of the umbilicus, and it extended beyond the median line into the right side of the abdomen. Fluctuation was very perceptible. Tenderness was excessive over the whole surface of the abdomen. It was found on examination that the tumour occupied but a small portion of the pelvis.

A few leeches and fomentations relieved the pain and tenderness; and until December, 1861, a period of three more years, very little change took place in either the tumour or her symptoms, except that she felt much better, although purulent matter had been found in the urine. She had passed some weeks at the sea-side in the summer, returning stronger and better in all respects. Several medical friends had seen her, who were all of opinion that it was a case of ovarian disease associated with hæmaturia.

In June, 1861, Mr. Spencer Wells saw her, and 
expressed the opinion that an ovarian cyst, or a pelvic abscess, communicated with the bladder, but discouraged a proposal to make any freer communication with the bladder or to inject iodine. The tumour remained almost unchanged in size or position until June, 1865, a period of four more years. At that time it began rapidly to increase, and on July 22nd, 1865, I was hastily summoned into Gloucestershire, where the patient was on a visit. There I met Mr. Child and Mr. H. Cooper; and finding the whole abdomen very tense and painful, and a prominent point situate equidistant between the crest of the ilium and the umbilicus on the left side, which was livid and the sac thin, I introduced a trocar and evacuated seven pints of sanguineous clotty fluid. The sac was very tough. A large mass remained in the abdomen, which appeared to be partly solid and partly multilocular. She continued very faint and sick for many hours after the operation, but all pain was relieved; and by degrees she so far recovered her strength as to be able, two years afterwards, to take moderate exercise, and even to play croquet for three or four hours at a time.

The opening made remained patent, giving vent to fetid discharge, which varied much in character and quantity from time to time, sometimes soaking through her clothes, at other times being very small in amount.

A year after the operation Mr. Spencer Wells saw her again. At that time the fistulous opening was situate close under the ribs, taking a downward course. The tumour was reduced to the size of a man's fist. The os uteri could not be felt, being dragged upwards by the tumour. Some of the fluid from the tumour Mr. Wells examined, but none of the elements of urine could be detected.

In June, 1867, some anasarca of the legs took place, and albumen was found in her urine. This symptom gradually increased, and in September, 1867, vomiting became very troublesome, and she very gradually sank on October 29th, retaining consciousness to the end.

Mr. Gay made a post-mortem examination on the following day, and gave me the accompanying notes : 
"Externally there was great fulness in the left lumbar region and the adjoining umbilical region in front. On percussing this space it was found to be dull as well in front as behind, whilst in the other regions of the abdomen percussion elicited a hollow sound. It was clear from a little manipulation that these physical signs were due to the presence below the integuments, which were moveable over it, of a large unyielding and somewhat irregularly oval-shaped body. There was a fistulous opening in the left hypochondrium, through which a probe was passed backwards and downwards in the direction of this tumour, prior to the abdomen being opened. On throwing back the integuments, this fistulous passage, the walls of which were very thick, was observed to lead to the supra-renal capsule, which together with the kidney formed the hard body alluded to. Together they formed a mass nearly six inches in its long, and above three inches in its short, diameter. The descending colon was closely adherent to it, but it had no other attachments in front, with the exception of that to the skin by means of the wall of the fistulous track just described. The mass was firmly bound down to the parts with which it was in contact behind, by close adhesion to the fascia over the atrophied quadratus lumborum muscle, and laterally by tough and thick fibrous bands which could only be severed by the scalpel. The surface of the mass was somewhat uneven, mottled, of a dark bluish and yellow colour, and still partially invested with fat. The kidney which was closely adherent to the supra-renal capsule formed a large cyst, from which, on cutting it through, a considerable quantity of thin ochry matter and shreds of bloody pus clot flowed. The tissues had become completely degenerated, the degeneration in all probability having begun with the membrane lining its pelvis and calyces. This membrane had become a thick, almost pulpy, dark greenish-coloured lamina, which appeared in the course of separating from its connections fragmentally; the tubular tissue had entirely disappeared, whilst the cortical was reduced to a very pale, thin stratum surrounded by the capsule, which in its turn had become very thick and almost 
of cartilaginous hardness and appearance. The ureter had degenerated to a hard cord and was entirely closed up. The emulgent artery and vein were large. The supra-renal capsule had been hollowed out by a large sinuous passage which formed a continuous channel from the fistulous track to the kidney. Its walls were dense and evidently lined with mucous membrane. Between them and the external tunic of the capsule there were here and there traces of the normal tissues. The adhesion of the capsule to the kidney was so firm that it could not be torn asunder. The right kidney was enlarged to twice its normal size, and was undergoing yellow degeneration.

The ovaries, lungs, liver, and spleen, were healthy."

Remarks.-It is worthy of observation that this tumour had existed for fifteen years, that it had commenced and was first observed in the situation of the left ovary, that no pain was ever referred to the region of the kidney, that the urine was normal except upon those occasions when she suffered from hæmaturia, and that the uterus was latterly drawn up so as to render it difficult or impossible to reach the os with the finger; that in the early stages of the disease the uterus was moveable and apparently normal in size, and that when some of the fluid from the tumour was examined no elements of urine could be detected. Of course the postmortem examination revealed the causes which produced these symptoms; and the total destruction of the secreting portion of the kidney accounts for the absence of urea in the abstracted fluid. With the exception of the hæmaturia, which naturally excited some doubt and question, there was nothing in the case which was not quite reconcileable with the diagnosis which had been formed of it by some of those most accustomed to the examination of this kind of cases. In fact, it became a question as to the propriety of removing the tumour should it become a source of much distress; and I well remember when I mentioned the case at a meeting of the Royal Medical and Chirurgical Society, after the reading of a paper by Dr. Lee on some cases of ovarian disease, that the fact of this complication of hæmaturia was not deemed 
by some Fellows who replied as a symptom which should interdict ovariotomy even should it arise from direct communication with the tumour.

A similar case to the one I have narrated is published by Mr. Spencer Wells in the 'Dublin Quarterly Journal of Medical Science' of February, 1867, in which ovariotomy was attempted with, of course, a fatal result. The more frequently difficulties and dangers are pointed out, the greater will be the success in practice, and I therefore deem this instructive case not unworthy of record. 